\title{
Ehrlichia ruminantium variants which do not cause heartwater found in South Africa
}

\author{
M.T.E.P. Allsopp ${ }^{a}$, M.F. Van Strijp ${ }^{a}$, E. Faber ${ }^{a}$, A.I. Josemans ${ }^{a}$ and B.A. Allsopp \\ ${ }^{\text {a} O n d e r s t e p o o r t ~ V e t e r i n a r y ~ I n s t i t u t e, ~ P r i v a t e ~ B a g ~ X 5, ~ O n d e r s t e p o o r t ~ 0110, ~ S o u t h ~ A f r i c a ~}$ \\ ${ }^{b}$ Department of Veterinary Tropical Diseases, Faculty of Veterinary Science, University \\ of Pretoria, Private Bag X04, Onderstepoort 0110, South Africa
}

\section{Abstract}

In 1994 a batch of apparently healthy goats was selected for intended export to the USA from a heartwater-free and vector tick-free region of South Africa. The animals were tested serologically for heartwater, using either or both an IFA and an ELISA test, and $52 \%$ were found to be serologically positive. A PCR assay based on Ehrlichia ruminantium $16 \mathrm{~S}$ gene sequences gave positive results for $54 \%$ of the animals, suggesting that apparently non-pathogenic E. ruminantium variants existed in this heartwater-free area. To identify and characterise the agents responsible for the positive serological and PCR results, ticks and animal blood samples were collected from two of the three farms involved in the original survey during two successive seasons of expected peak tick activity. Ticks were kept alive for a minimum of 3 weeks to allow digestion of any blood meal before being processed.

Over the two seasons, $28 \%$ of the livestock and $15 \%$ of the ticks sampled were found to be carrying E. ruminantium. E. ruminantium $16 \mathrm{~S}$ and pCS20 sequences were detected in all of the four tick species collected from the livestock (Rhipicephalus evertsi evertsi, Rhipicephalus evertsi mimeticus, Hyalomma truncatum, Hyalomma marginatum rufipes), suggesting that some of the species may act as vectors. Animals generally carried multiple E. ruminantium $16 \mathrm{~S}$ genotypes, whereas ticks rarely carried more than one. Infection levels in both animals and ticks were too low to generate a marked response 
when a blood stabilate was sub-passaged in a clean sheep, preventing the subsequent establishment of any of the organisms in culture.

\section{Article Outline}

1. Introduction

2. Materials and methods

3. Results

3.1. Animal and tick samples

3.2. $\mathrm{pCS} 20$ and $16 \mathrm{~S}$ probing

3.3. pCS20 and $16 \mathrm{~S}$ genotype sequence analysis

3.4. Sequences from amplicons of unexpected sizes

3.5. Attempted transmission from blood stabilate to clean sheep

4. Discussion

5. Conclusion

Acknowledgements

References

\section{Introduction}

Heartwater is a disease of ruminants caused by the intracellular rickettsia Ehrlichia ruminantium (previously Cowdria ruminantium) in sub-Saharan Africa. The disease is only transmitted by ticks of the genus Amblyomma and its distribution coincides with that of its vector species (Walker and Olwage, 1987). Heartwater is a major obstacle to domestic livestock productivity in areas where it is endemic (Mukhebi et al., 1999), and is particularly serious when susceptible animals are moved from heartwater-free to heartwater-infected areas (Simpson et al., 1987). Amblyomma variegatum, the most widespread African vector of heartwater, was introduced, together with the disease, on to some Caribbean islands during the 18th or 19th centuries (Maillard and Maillard, 1998). It is possible that the disease could spread from the Antillean islands to the American mainland (Deem, 1998), where a competent tick vector is already present (Mahan et al., 2000), and this would have severe consequences for the cattle industry in the Americas. 
In 1994, Boergoats from three farms in the Groblershoop district of the northern Cape Province of South Africa, a region free of heartwater and of the only South African heartwater vector tick, Amblyomma hebraeum, were held in quarantine in a facility near Cape Town prior to shipment to the USA. The goats were screened for heartwater using the ELISA and IFA tests available at the time (Du Plessis et al., 1993) and 52\% of the animals tested positive for heartwater by either or both of the tests. These animals were not exported. A PCR assay which could detect five different E. ruminantium 16S genotypes gave positive results for $54 \%$ of the animals, suggesting that apparently nonpathogenic E. ruminantium variants existed in this heartwater-free area (Allsopp et al., 1997). One farm (Morgenswag) had contributed 47 animals, of which one was seropositive by ELISA, 19 were IFA positive, and 43 (91\%) were probe positive for one or more of the E. ruminantium 16S genotypes.

We decided to try to further characterise, and if possible establish in culture, the organism(s) which gave rise to the confusing serological and PCR results in 1994 in order to improve the dependability of a "positive heartwater" diagnosis. We returned to the same area of the Northern Cape and collected blood samples and ticks from cattle, sheep and goats from two farms, Morgenswag and Doktershoek, for sequence investigation.

\section{Materials and methods}

Blood (10 ml aliquots in EDTA) and ticks were collected from cattle and sheep on the farm Morgenswag $\left(28^{\circ} 32^{\prime} \mathrm{S}, 2^{\circ} 27^{\prime} \mathrm{E}\right)$ and from goats on the farm Doktershoek $\left(28^{\circ} 34^{\prime} \mathrm{S}\right.$, $\left.22^{\circ} 29^{\prime} \mathrm{E}\right)$. Blood stabilates were prepared in situ as follows: equal volumes of blood and SPG (sucrose: $74.62 \mathrm{~g} / 1, \mathrm{KH}_{2} \mathrm{PO}_{4}: 0.517 \mathrm{~g} / 1, \mathrm{~K}_{2} \mathrm{HPO}_{4}: 1.643 \mathrm{~g} / \mathrm{l}$, K-glutamate: $0.907 \mathrm{~g} / \mathrm{l}$ ) were mixed and sealed in $\sim 5 \mathrm{ml}$ aliquots after separation of $200 \mu \mathrm{l}$ for DNA extraction. Stabilates were frozen immediately in liquid nitrogen for attempted infection studies. The ticks were maintained alive at $27 \pm 2{ }^{\circ} \mathrm{C}$ and $75-80 \%$ humidity for between 3 weeks and 3 months after identification. Individual ticks were then washed in ethanol and air dried before maceration in SPG (1 ml per tick). The resulting stabilates were stored in aliquots in liquid nitrogen, after separation of $200 \mu \mathrm{l}$ for DNA extraction.

DNA was extracted from $200 \mu \mathrm{l}$ aliquots of blood stabilates using the GENTRA 'Generation Capture Column' system (Gentra Corp. Minnesota, MN, USA) according to 
the manufacturer's instructions and was eluted from the columns with $100 \mu$ l elution buffer. DNA was extracted from tick stabilates $(200 \mu \mathrm{l})$ using the QIAamp DNA mini kit (QIAGEN, Hilden, Germany) according to the manufacturer's protocol, except that proteinase $\mathrm{K}$ digestion was carried out at $56{ }^{\circ} \mathrm{C}$ overnight. DNA was eluted in $100 \mu \mathrm{l}$ of the elution buffer supplied with the kit. DNA samples were stored at $-20{ }^{\circ} \mathrm{C}$ pending analysis.

Five microlitre aliquots of DNA were subjected to a PCR using primers HH1-F and HH2$\mathrm{R}$ for amplification of the pCS20 region (Van Heerden et al., 2004). A 'no-DNA' (water) negative control and an E. ruminantium (Welgevonden) (Du Plessis, 1985) tissue culture DNA positive control were amplified at the same time. Aliquots $(5 \mu \mathrm{l})$ of each PCR product were run on 1\% agarose/TBE gels with phiX174/HaeIII markers to check for visible amplicons. Aliquots $(10 \mu \mathrm{l})$ of each PCR product were slot blotted and probed as described (Allsopp et al., 1997). Samples giving strong hybridisation signals with the pCS20 probe were selected for pCS20 and 16S V1 loop amplification and sequencing. To obtain visible pCS20 products, a nested PCR using primer pair AB128/AB127 (Mahan et al., 1992) was carried out using $1 \mu \mathrm{l}$ aliquots of the primary (HH1-F/HH2-R) amplicons. DNA samples positive for pCS20 were subjected to 16S V1 loop amplification and probing to determine the $16 \mathrm{~S}$ genotypes present.

At least two PCR amplifications were carried out for each of the selected pCS20-positive samples and amplicons were pooled before cloning and sequencing to minimise the effects of polymerase misincorporation errors. Where multiple bands were observed PCR pools were gel-purified. Amplicons (pCS20, $903 \mathrm{bp} ; 16 \mathrm{~S}, 328 \mathrm{bp}$ ) of the expected size were excised and extracted from gel slices using the MinElute PCR purification kit gel extraction protocol (Qiagen, Hilden, Germany). Some representative examples of bands of unexpected sizes were also purified. Purified amplicons were cloned into pGEM-T vector (Promega, Madison, USA) according to the manufacturer's protocol. Bacterial colonies positive for E. ruminantium were identified from colony lifts on nylon filters (Magna Lift, Osmonics Inc., Minnetonka, USA) by probing with a ${ }^{32} \mathrm{P}$-labelled oligonucleotide which hybridizes to all five E. ruminantium 16S V1 loop sequences (Allsopp et al., 1997) or with a ${ }^{32} \mathrm{P}$-labelled random primed fragment of the Welgevonden pCS20 region (Van Heerden et al., 2004). In the case of cloned amplicons of unexpected 
sizes white colonies were picked at random. Picked colonies were grown overnight in $3 \mathrm{ml}$ aliquots of LB medium with tetracycline and ampicillin as recommended in the Promega protocol and plasmid preparation was carried out using the High Pure Plasmid Isolation kit (Roche Diagnostics, Mannheim, Germany). Plasmid sequencing was carried out using 'big dye' chemistry on an ABI 3100 automated sequencer. 16S and pCS20 sequences were compared with existing E. ruminantium databases using the 'Seqlab' module of the Wisconsin Package version 10.1 (Genetics Computer Group, Madison, WI, USA). BLAST searches of GenBank were conducted with sequences from amplicons of unexpected sizes.

An aliquot $(10 \mathrm{ml})$ of blood stabilate from one pCS20-positive sheep (sheep MS4) was injected intravenously into one pCS20-negative sheep (sheep S171) to check for pathogenicity. The temperature of the animal was monitored daily and blood was collected for attempted culture at peak temperature increase. DNA was extracted from a $200 \mu \mathrm{l}$ blood aliquot as described and 16S amplicons were cloned and sequenced. Direct sequencing of the pCS20 amplicon was carried out.

All E. ruminantium sequences were submitted to GenBank and were assigned the following accession numbers:

16S: DQ640389-DQ640401; pCS20: DQ631917-DQ631931.

\section{Results}

\subsection{Animal and tick samples}

In the two seasons surveyed, the number of animals from which blood samples and ticks were collected are listed in Table 1, together with the numbers of animals testing pCS20 positive. A total of 106 ticks were collected from the animals in 2002, and 116 in 2003. The numbers and identities of the ticks, together with the numbers of ticks pCS20 positive, are given in Table 2. More ticks were collected from cattle in 2002 than in 2003, when only 16 were collected, probably because the animals had been dipped shortly before the survey. All 16 were pCS20 negative. The pCS20 probing results for animals and ticks sampled in 2003 are shown in Fig. 1.

Table 1. 
Animals on two Northern Cape farms from which blood samples were collected in 2002 and 2003: total number of samples and numbers testing positive with the pCS20 probe

\begin{tabular}{|l|l|l|l|l|}
\hline Host & \multicolumn{2}{|l|}{2002} & \multicolumn{2}{l|}{2003} \\
\hline & Total & pCS20 +ve & Total & pCS20 +ve \\
\hline Cattle & 7 & 3 & 10 & 2 \\
\hline Sheep & 8 & 2 & 10 & 3 \\
\hline Goats & 6 & 2 & 10 & 3 \\
\hline
\end{tabular}

Table 2.

Tick species collected from animals on two Northern Cape farms during 2002 and 2003: total number of ticks collected and numbers testing positive with the $\mathrm{pCS} 20$ probe

\begin{tabular}{|c|c|c|c|c|c|c|c|c|}
\hline \multirow[t]{2}{*}{$\begin{array}{l}\text { Host and ticks testing } \\
\text { pCS20 + ve }\end{array}$} & \multicolumn{4}{|c|}{$\begin{array}{l}2002 \text { tick species and } \\
\text { number }\end{array}$} & \multicolumn{4}{|c|}{$\begin{array}{l}2003 \text { tick species and } \\
\text { number }\end{array}$} \\
\hline & Ree & Rem & Ht & Hmr & Ree & Rem & Ht & Hmr \\
\hline Cattle & 1 & 35 & 0 & 3 & 0 & 10 & 0 & 6 \\
\hline Sheep & 0 & 31 & 2 & 0 & 4 & 35 & 5 & 12 \\
\hline Goats & 34 & 0 & 0 & 0 & 44 & 0 & 0 & 0 \\
\hline Total ticks & \multicolumn{4}{|l|}{106} & \multicolumn{4}{|l|}{116} \\
\hline pCS20+ & 2 & 9 & 0 & 0 & 1 & 4 & 1 & 5 \\
\hline
\end{tabular}

Key: Ree, Rhipicephalus evertsi evertsi; Rem, Rhipicephalus evertsi mimeticus; Ht, Hyalomma truncatum; Hmr, Hyalomma marginatum rufipes. 


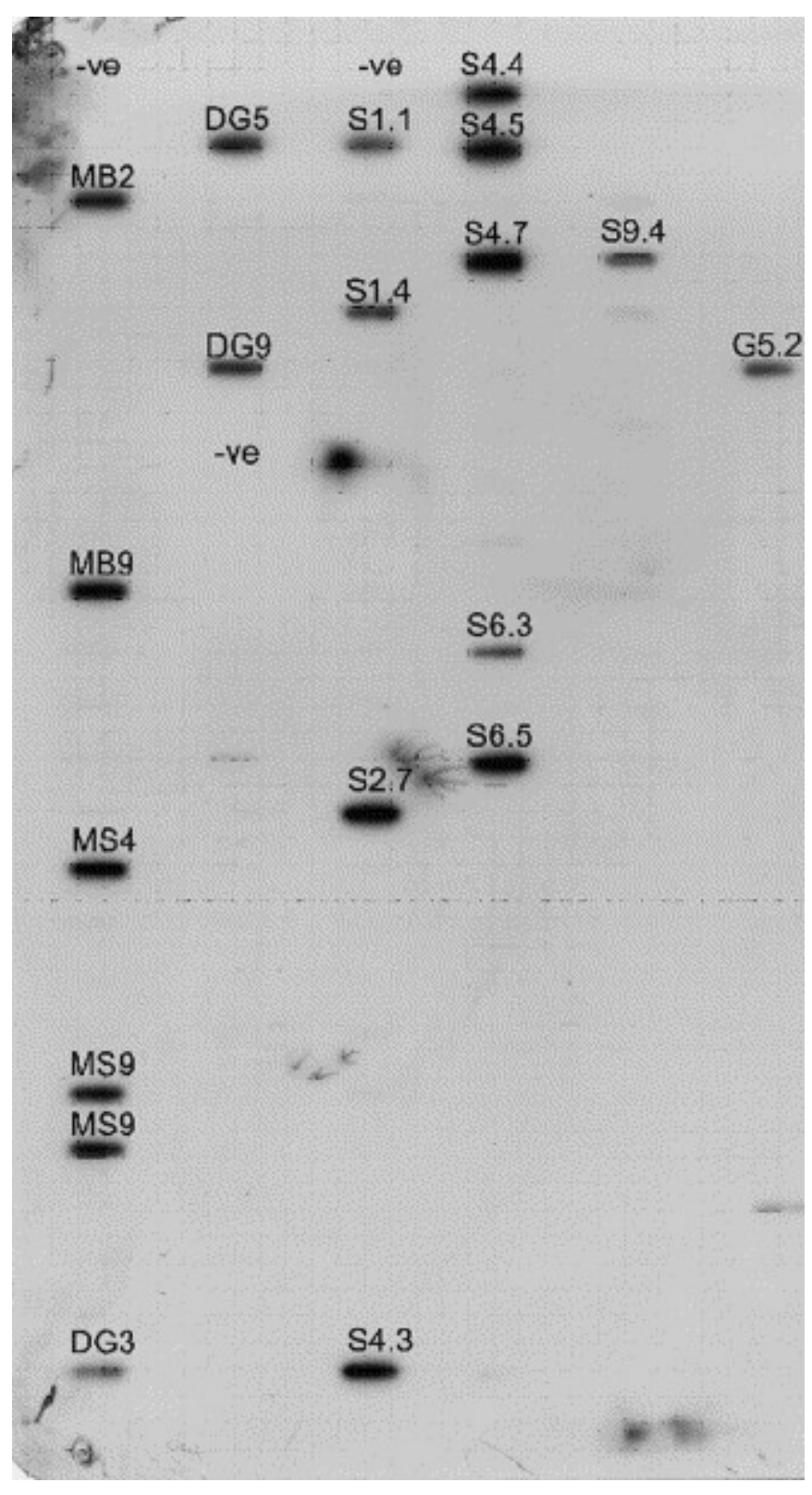

Fig. 1. Autoradiograph showing pCS20 probe results for animals and ticks collected in 2003.

Only $R$. evertsi evertsi ticks were collected from the goats on Doktershoek farm in both 2002 and 2003. The sheep and cattle on Morgenswag were infested predominantly with $R$. evertsi mimeticus, a sub-species closely related to $R$. evertsi evertsi, and some animals on this farm were also carrying $H$. truncatum and $H$. marginatum rufipes ticks. We found only these four tick species, and $9.8 \%$ of them tested positive for E. ruminantium using the pCS20 assay. 


\section{2. pCS20 and $16 S$ probing}

In 2002 the only ticks found to be pCS20 positive were of the two Rhipicephalus spp., whereas in 2003 all four species yielded pCS20-positive individuals (Table 2). We selected animals and ticks from 2003 for sequence analysis, in order to obtain better coverage of the E. ruminantium genotypes present, since it was possible that the different genotypes might be preferentially carried by different tick species.

In 2003 all the animals appeared to be carrying more than one E. ruminantium 16S genotype (Table 3), whereas the ticks rarely carried more than one. The Ball 3 genotype was detected in one animal and all the animals appeared to be carrying the Omatjenne 16S genotype. Only one tick (H. marginatum rufipes) gave a faint positive signal with the Omatjenne genotype probe.

Table 3.

Results obtained with five different Ehrlichia ruminantium 16S probes used to test all pCS20-positive blood and tick samples

\begin{tabular}{|c|c|c|c|c|c|c|}
\hline \multirow{2}{*}{$\begin{array}{l}\text { Animal or tick } \\
\text { ID }\end{array}$} & \multirow[t]{2}{*}{ Species } & \multicolumn{5}{|c|}{ 16S V1 loop probe } \\
\hline & & $\begin{array}{l}\text { Ball } \\
3\end{array}$ & Senegal & Omatjenne & $\begin{array}{l}\text { Mara } \\
87 / 7\end{array}$ & Welgevonden \\
\hline MB1 & Bovine & - & + & + & + & + \\
\hline MB9 & Bovine & + & $?+$ & + & + & + \\
\hline MS4 & Ovine & - & + & + & + & + \\
\hline MS9 & Ovine & - & + & + & + & + \\
\hline DG2 & Caprine & - & - & + & + & + \\
\hline DG3 & Caprine & - & + & + & + & + \\
\hline DG5 & Caprine & - & + & + & + & + \\
\hline MS1.1 & $\mathrm{Hmr}$ & - & + & - & - & - \\
\hline MS1.4 & $\mathrm{Hmr}$ & - & + & - & - & - \\
\hline MS2.7 & Rem & - & + & - & - & - \\
\hline
\end{tabular}




\begin{tabular}{|l|l|l|l|l|l|l|}
\hline $\begin{array}{l}\text { Animal or tick } \\
\text { ID }\end{array}$ & Species & \multicolumn{7}{|l|}{ 16S V1 loop probe } \\
\hline & & $\begin{array}{l}\text { Ball } \\
\mathbf{3}\end{array}$ & Senegal & Omatjenne & $\begin{array}{l}\text { Mara } \\
87 / 7\end{array}$ & Welgevonden \\
\hline MS4.3 & Hmr & - & + & - & + & + \\
\hline MS4.4 & Hmr & - & - & - & + & - \\
\hline MS4.5 & Hmr & - & - & $?+$ & + & - \\
\hline MS4.7 & Rem & - & + & - & + & - \\
\hline MS6.5 & Rem & - & - & - & + & + \\
\hline MS9.4 & Ht & - & - & - & + & - \\
\hline DG5.2 & Ree & - & - & - & + & - \\
\hline
\end{tabular}

Ree, $R$. evertsi evertsi; Rem, $R$. evertsi mimeticus; Ht, H. truncatum; Hmr, $H$. marginatum rufipes; (+) positive signal; (-) no signal observed; (?+) very faint signal, possibly negative.

\section{3. pCS20 and $16 \mathrm{~S}$ genotype sequence analysis}

The sequence results are presented in Table 4. pCS20 sequences identical to those of $E$. ruminantium strains Welgevonden and Kiswani were detected in the bovine MB9, the same Kiswani pCS20 sequence was also detected in the R. evertsi evertsi tick DG5.2 recovered from goat DG5. The pCS20 sequence detected in goat DG5 was identical to that of E. ruminantium (Umbanein) and three new unique pCS20 sequences were also obtained, two from sheep MS9 and one from sheep MS4. In contrast, most of the 16S sequences were of the Senegal, Mara 87/7 and Welgevonden genotypes, although one previously unknown sequence was also obtained. In the case of the ticks, there was no correlation between their species and the E. ruminantium sequences which they carried. Although the probing results showed that all the animals appeared to be carrying the $E$. ruminantium (Omatjenne) genotype, no Omatjenne 16S sequences were obtained. We were also unable to obtain a Ball $316 \mathrm{~S}$ sequence from the one animal in which that genotype had been detected by probing. 
Table 4.

Genotype identities of different pCS20 sequences, and genotype identities of different 16S sequences, obtained from pCS20-positive blood and tick samples

\begin{tabular}{|l|l|l|l|}
\hline $\begin{array}{l}\text { Animal/tick } \\
\text { ID }\end{array}$ & Species & pCS20 seqs & 16S genotypes \\
\hline MB9 & Bovine & $\begin{array}{l}\text { Welgevonden, } \\
\text { Kiswani }\end{array}$ & $\begin{array}{l}\text { Senegal, Welgevonden, Mara 87/7, } \\
\text { new }\end{array}$ \\
\hline MS4 & Sheep & 1 unique & Welgevonden \\
\hline MS4.3 & $\begin{array}{l}\text { Hmr } \\
\text { tick }\end{array}$ & No amplicon & Mara 87/7 \\
\hline MS4.7 & Rem & No amplicon & Senegal \\
\hline MS9 & Sheep & 2 unique & Senegal, Mara 87/7 \\
\hline MS9.4 & Ht tick & No amplicon & Mara 87/7 \\
\hline DG5 & Goat & Umbanein & Senegal \\
\hline DG5.2 & Ree tick & Kiswani & Welgevonden, Mara 87/7 \\
\hline
\end{tabular}

Ree, $R$. evertsi evertsi; Rem, $R$. evertsi mimeticus; Ht, $H$. truncatum; Hmr, $H$. marginatum rufipes.

\subsection{Sequences from amplicons of unexpected sizes}

Most of the amplicons of unexpected sizes were much less prominent than the $16 \mathrm{~S}$ and pCS20 amplicons. All those from which sequences were obtained were found to match mammalian chromosomal sequences in GenBank.

\subsection{Attempted transmission from blood stabilate to clean sheep}

When blood stabilate from sheep MS4 was injected into sheep S171, the animal reacted 7 days post-inoculation with a temperature of $40.3{ }^{\circ} \mathrm{C}$, but showed no signs of distress. The temperature fluctuated thereafter, reaching a maximum of $40.4^{\circ} \mathrm{C} 30$ days postinoculation, after which no further records were made. No Ehrlichia organisms were observed in blood smears and the levels of infection in the animal were not sufficient for the establishment of cultures. Amplification of a blood sample taken at day 26 post- 
inoculation (temperature $40.3^{\circ} \mathrm{C}$ ) and probing for pCS20 indicated that E. ruminantium was present. One 16S V1 loop sequence identical to that of E. ruminantium (Welgevonden) was obtained from this animal, but pCS20 sequencing indicated that a mixture of genotypes was present.

\section{Discussion}

The two farms, Doktershoek and Morgenswag, are situated on opposite sides of a road, close to a nature reserve. Four species of ticks were found on Morgenswag farm, $R$. evertsi evertsi, $R$. evertsi mimeticus, $H$. truncatum and $H$. marginatum rufipes, with the Hyalomma ticks being present in much smaller numbers than the Rhipicephalus species. Hyalomma ticks were not found at all on Doktershoek, possibly because fewer animals were sampled, and because these ticks are more restricted in their feeding preferences than Rhipicephalus species (I.G. Horak, personal communication). R. evertsi mimeticus ticks are not widespread in South Africa, but they are common in Angola and Namibia and are known to have been introduced into some sites in South Africa, presumably with imported livestock (Walker et al., 2003). The sub-species has obviously become well established on Morgenswag farm.

Diagnostic tests targeting the pCS20 region have long been considered to be specific for E. ruminantium (Allsopp et al., 1999), and the sequences of this region from 14 different virulent heartwater-producing isolates have been shown to be phylogenetically closely related, and much more divergent from the sequence of the homologous region of Ehrlichia chaffeensis (Van Heerden et al., 2004). Three of the pCS20 sequences obtained in this study were identical to those from known virulent heartwater-producing $E$. ruminantium isolates Welgevonden, Kiswani and Umbanein, and three were unique (Table 4). The new unique sequences were closely similar to the others, exhibiting only small numbers of SNPs, and they fell firmly into the E. ruminantium clade on phylogenetic analysis (data not shown). All except one of the $16 \mathrm{~S}$ sequences we obtained were identical over the sequenced region to those of the well-characterised pathogenic $E$. ruminantium isolates Welgevonden, Senegal and Mara 87/7 found in heartwater-endemic areas. The new unique sequence was closely similar to the others, and it too fell firmly into the E. ruminantium clade on phylogenetic analysis (data not shown). No heartwater 
disease is ever seen in this area of the Northern Cape, so we must assume that, despite sharing common pCS20 and $16 \mathrm{~S}$ sequences, these E. ruminantium organisms differ markedly from pathogenic isolates in both infectivity and virulence. Infection levels in both animals and ticks also appear to be very low, as shown by our inability to obtain sequences from some samples which gave positive probe results.

It has been known for some years that at least one E. ruminantium genotype exists which does not cause heartwater in its area of origin, this is E. ruminantium (Omatjenne), obtained from a $H$. truncatum tick in a heartwater-free area of Namibia (Du Plessis, 1990). In the molecular probing survey carried out in 1994, and referred to in the introduction, 166 animals from the heartwater-free Northern Cape were examined. Seventy animals (42\%) were probe positive for the Omatjenne 16S genotype (Allsopp et al., 1997) while 40 animals (24\%) gave probe signals for $16 \mathrm{~S}$ sequences from the pathogenic E. ruminantium genotypes Ball 3 and Welgevonden. In the present survey all seven E. ruminantium-positive animals gave a good signal with the Omatjenne 16S probe but only one $H$. marginatum rufipes tick gave a faint signal with this probe, suggestive of a very low infection rate.

Both $H$. truncatum and $H$. marginatum rufipes are two-host ticks, with the larvae and nymphs feeding exclusively on small animals and the adults feeding exclusively on large mammals. The immature stages of $H$. truncatum feed on scrub hares (Lepus saxatilis) and rodents, while the immature stages of $H$. marginatum rufipes feed on scrub hares and birds (I.G. Horak, personal communication). This means that ticks of the genus Hyalomma can only be vectors for E. ruminantium if larvae are infected transovarially or if adults transmit the infection intrastadially. Apart from a single unconfirmed report (Bezuidenhout and Jacobsz, 1986) it is not generally believed that E. ruminantium undergoes transovarial transmission, and intrastadial infection via Hyalomma adults would only occur in the event of accidental dislodgement of a partially fed tick, followed by re-attachment to a new host. Hyalomma ticks are therefore unlikely to be anything other than occasional and accidental vectors of E. ruminantium. E. ruminantium (Omatjenne) was originally obtained from an adult female $H$. truncatum tick taken from a bovine, triturated, and injected into a mouse (Du Plessis, 1990). This tick could well have 
infected the mouse simply because it was carrying infected bovine blood, rather than because it was an active vector of E. ruminantium.

$R$. evertsi spp. are also two-host ticks but both immature and adult stages may infest large mammalian hosts, as well as scrub hares and small antelopes. The failure to detect $E$. ruminantium (Omatjenne) in any of the Rhipicephalus ticks suggests that this organism does not survive digestion of the blood meal in ticks of this genus. It is known that pathogenic strains of E. ruminantium can be transmitted by the tortoise tick, Amblyomma marmoreum (Peter et al., 2000), which is present in this area of the Northern Cape, so it is possible that this tick is a vector for less virulent E. ruminantium variants such as the Omatjenne strain. The cattle, sheep and goats in this study roam freely in the bush, hence we cannot discount the possibility that they may become infected, via these ticks, by any of the E. ruminantium variants we detected. Nevertheless, field collections of $A$. marmoreum from domestic animals are rare (Walker and Olwage, 1987) and none were recovered from the animals in our study. As for the Rhipicephalus species in which the $E$. ruminantium variants were detected, further work is needed to determine whether they are competent vectors for transmission of the organisms.

Positive serological results from heartwater-free areas cannot simply be ignored, but they also cannot be assumed to indicate the presence of potentially heartwater-causing organisms. Even the most sensitive and specific ELISA for E. ruminantium (MAP1B) gives positive cross reactions with E. chaffeensis and Ehrlichia canis sera (van Vliet et al., 1995), and while there is no molecular evidence that E. chaffeensis occurs in southern Africa (MTEPA, unpublished), and we have never detected E. canis $16 \mathrm{~S}$ sequences in ruminants or in ticks recovered from ruminants, other uncharacterised Ehrlichia spp. could be a source of positive MAP1B reactions. In earlier work, for example, a 16S sequence (GenBank accession number U54805) closely similar to E. ovina (GenBank accession number AF318946.1) was obtained from a sheep on the farm Germishuys (Allsopp et al., 1997). It likely, however, that positive MAP1B results from heartwaterfree areas, particularly if the animals are also pCS20 positive, indicates exposure to $E$. ruminantium of low pathogenicity. In the present work we were unable to obtain visible pCS20 amplicons from three of the ticks from which E. ruminantium 16S sequences were obtained. This is likely to be the result of polymorphisms in one or both of the pCS20 
nested amplification primer target sites preventing effective hybridization and chain extension. It is also possible that serologically positive but pCS20-negative animals could be carrying other uncharacterised Ehrlichia spp. To summarize: positive "heartwater" serology in heartwater-free areas of southern Africa probably indicates exposure to $E$. ruminantium of low pathogenicity or to other uncharacterised Ehrlichia spp. The organisms may be unable to cause heartwater disease, but the animals involved should not be exported to heartwater-free regions, especially not to those where there are Amblyomma ticks capable of transmitting E. ruminantium. The danger that the virulence of the organisms could be affected by the tick host, as suggested by Du Plessis et al. (1993), has never been properly investigated, and such investigations must await establishment of the variant organisms in culture.

The data presented in Table 2 represent only adult ticks which survived for periods of 3 weeks to 3 months after collection. Detection of E. ruminantium in these ticks thus indicates that viable organisms were still present long after digestion of the blood meal. The detection of single E. ruminantium genotypes in ticks recovered from animals carrying multiple genotypes may be due to infection exclusion, an effect which has been shown to occur in Dermacentor variabilis ticks fed on animals infected with different strains of Anaplasma marginale (de la Fuente et al., 2003). The relatively high percentage of infection (10.5\%) in the surviving Rhipicephalus spp. ticks suggests that these ticks may act as vectors for the transmission of apparently non-pathogenic E. ruminantium variants. This possibility was proposed as long ago as 1993 for both $H$. truncatum and $R$. evertsi (Du Plessis, 1993), but for the reasons stated in an earlier paragraph, Hyalomma spp. ticks are unlikely to be vectors. Du Plessis ascribed positive heartwater serological results in heartwater-free areas to uncharacterised Ehrlichia species, as opposed to what was then known as C. ruminantium, and the molecular sequence data obtained during this study represent a first step in the characterisation of the organisms. Infection levels in animals and ticks in the field are very low and we failed to induce a level of infection by blood stabilate passage into a clean sheep sufficient to initiate a culture. 


\section{Conclusion}

It appears that there are many different E. ruminantium genotypes in circulation, with pathogenicities varying from high to low, and that pathogenicity does not correlate with either pCS20 or $16 \mathrm{~S}$ genotype. Further molecular genetic characterisation, and transmission and pathogenicity studies, of the low pathogenicity genotypes must wait until the organisms are established in culture. As other workers have observed, a bacterial sample principally composed of disease-causing isolates is highly biased and represents a minor (and atypical) fraction of a much larger and more diverse population (Feil and Spratt, 2001).

\section{References}

Allsopp et al., 1999 M.T. Allsopp, C.M. Hattingh, S.W. Vogel and B.A. Allsopp, Evaluation of 16S, map1 and pCS20 probes for detection of Cowdria and Ehrlichia species, Epidemiol. Infect. 122 (1999), pp. 323-328.

Allsopp et al., 1997 M.T. Allsopp, E.S. Visser, J.L. Du Plessis, S.W. Vogel and B.A. Allsopp, Different organisms associated with heartwater as shown by analysis of $16 \mathrm{~S}$ ribosomal RNA gene sequences, Vet. Parasitol. 71 (1997), pp. 283-300. Bezuidenhout and Jacobsz, 1986 J.D. Bezuidenhout and C.J. Jacobsz, Proof of transovarial transmission of Cowdria ruminantium by Amblyomma herbraeum, Onderstepoort J. Vet. Res. 53 (1986), pp. 31-34.

de la Fuente et al., 2003 J. de la Fuente, E.F. Blouin and K.M. Kocan, Infection exclusion of the rickettsial pathogen Anaplasma marginale in the tick vector Dermacentor variabilis, Clin. Diagn. Lab. Immunol. 10 (2003), pp. 182-184.

Deem, 1998 S.L. Deem, A review of heartwater and the threat of introduction of Cowdria ruminantium and Amblyomma spp. ticks to the American mainland, J. Zoo Wildl. Med. 29 (1998), pp. 109-113.

Du Plessis, 1985 J.L. Du Plessis, A method for determining the Cowdria ruminantium infection rate of Amblyomma hebraeum: effects in mice injected with tick homogenates, Onderstepoort J. Vet. Res. 52 (1985), pp. 55-61. 
Du Plessis, 1990 J.L. Du Plessis, Increased pathogenicity of an Ehrlichia-like agent after passage through Amblyomma hebraeum: a preliminary report, Onderstepoort J. Vet. Res. 57 (1990), pp. 233-237.

Du Plessis, 1993 J.L. Du Plessis, The relationship between Cowdria and Ehrlichia: change in the behaviour of ehrlichial agents passaged through Amblyomma hebraeum, Rev. Elev. Med. Vet. Pays. Trop. 46 (1993), pp. 131-143.

Du Plessis et al., 1993 J.L. Du Plessis, J.D. Bezuidenhout, M.S. Brett, E. Camus, F. Jongejan, S.M. Mahan and D. Martinez, The sero-diagnosis of heartwater: a comparison of five tests, Rev. Elev. Med. Vet. Pays. Trop. 46 (1993), pp. 123-129.

Feil and Spratt, 2001 E.J. Feil and B.G. Spratt, Recombination and the population structures of bacterial pathogens, Annu. Rev. Microbiol. 55 (2001), pp. 561-590. Mahan et al., 2000 S.M. Mahan, T.F. Peter, B.H. Simbi, K. Kocan, E. Camus, A.F. Barbet and M.J. Burridge, Comparison of efficacy of American and African Amblyomma ticks as vectors of heartwater (Cowdria ruminantium) infection by molecular analyses and transmission trials, J. Parasitol. 86 (2000), pp. 44-49. Mahan et al., 1992 S.M. Mahan, S.D. Waghela, T.C. McGuire, F.R. Rurangirwa, L.A. Wassink and A.F. Barbet, A cloned DNA probe for Cowdria ruminantium hybridizes with eight heartwater strains and detects infected sheep, J. Clin. Microbiol. 30 (1992), pp. 981-986.

Maillard and Maillard, 1998 J.C. Maillard and N. Maillard, Historique du peuplement bovin et de l'introduction de la tique Amblyomma variegatum dans les îles françaises des Antilles: synthèse bibliographique, Ethnozootechnie 1 (1998), pp. 19-36.

Mukhebi et al., 1999 A.W. Mukhebi, T. Chamboko, C.J. O’Callaghan, T.F. Peter, R.L. Kruska, G.F. Medley, S.M. Mahan and B.D. Perry, An assessment of the economic impact of heartwater (Cowdria ruminantium infection) and its control in Zimbabwe, Prev. Vet. Med. 39 (1999), pp. 173-189.

Peter et al., 2000 T.F. Peter, M.J. Burridge and S.M. Mahan, Competence of the African tortoise tick, Amblyomma marmoreum (Acari: Ixodidae), as a vector of the agent of heartwater (Cowdria ruminantium), J. Parasitol. 86 (2000), pp. 438-441. Simpson et al., 1987 B.C. Simpson, M.S. Lindsay, J.R. Morris, F.S. Muirhead, A. Pollock, S.G. Prichard, H.G. Stanley, G.R. Thirlwell, A.G. Hunter and J. Bradley, 
Protection of cattle against heartwater in Botswana: comparative efficacy of different methods against natural and blood-derived challenges, Vet. Rec. 120 (1987), pp. 135138.

Van Heerden et al., 2004 H. Van Heerden, H.C. Steyn, M.T. Allsopp, E. Zweygarth, A.I. Josemans and B.A. Allsopp, Characterization of the pCS20 region of different Ehrlichia ruminantium isolates, Vet. Microbiol. 101 (2004), pp. 279-291.

van Vliet et al., 1995 A.H. van Vliet, B.A. van der Zeijst, E. Camus, S.M. Mahan, D. Martinez and F. Jongejan, Use of a specific immunogenic region on the Cowdria ruminantium MAP1 protein in a serological assay, J. Clin. Microbiol. 33 (1995), pp. 2405-2410.

Walker et al., 2003 Walker, A.R., Bouattour, A., Camicas, J.-L., Estrada-Peña, A., Horak, I.G., Latif, A.A., Pegram, R.G., Preston, P.M., 2003. Ticks of Domestic Animals in Africa: A Guide to Identification of Species. Bioscience Reports. 43 Comiston Drive, Edinburgh EH10 5QR, UK, p. 171.

Walker and Olwage, 1987 J.B. Walker and A. Olwage, The tick vectors of Cowdria ruminantium (Ixodoidea, Ixodidae, genus Amblyomma) and their distribution, Onderstepoort J. Vet. Res. 54 (1987), pp. 353-379.

Corresponding author. Tel.: +27 12529 8426; fax: +27 125298312 . 